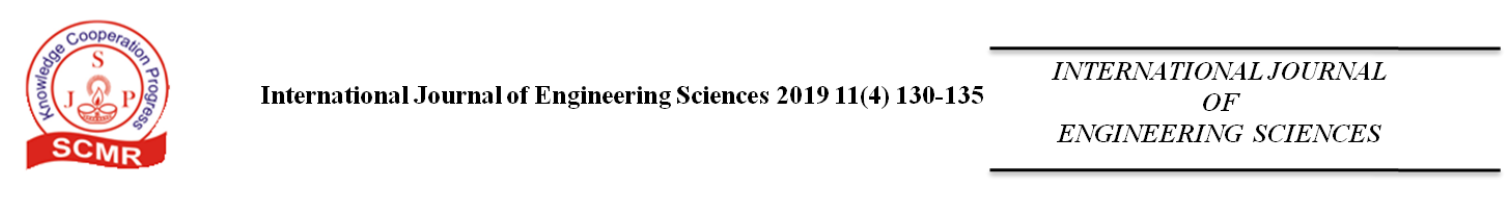

\title{
The Inference System for Fault Diagnosis
}

\author{
Yongeui Hong*, Taewoo Lee", Kyoungdyuk Rho", Hyun-Seung Cha ${ }^{\$}$, Jonghyo Lee ${ }^{\&}$ \\ *,\#Artillery System Team, Hanwha Land System R\&D Center, Korea \\ ${ }^{\$}$ SeoulStandard Co. Ltd., Korea \\ \&Agency for Defense Development, Korea \\ (Received 10 June 2018; accepted 15 October 2018)
}

https://doi.org/10.36224/ijes.110402

\begin{abstract}
A knowledge-based fault diagnosis system uses prior knowledge and context knowledge for prediction to improve fault diagnosis performance. The proposed representative fault diagnosis system consists of three levels. With this structure, fault diagnosis can be flexibly performed even in a complicated environment. The three-level consists of the fault diagnosis level, the learning level, and the information processing level. The Fault diagnosis level is able to express the correlation by using the data obtained from the controller and diagnose the fault by logically inferring it. The learning level links the logical language perceived by humans and the numerical data processed by the computer, and keeps it consistent with the situation. The information processing level acquires the feature value required by the higher level in the candidate region among the numerous data obtained from the controller and sends it to the higher level. The proposed algorithm can effectively diagnosis faults by using additional prior knowledge and situation data.
\end{abstract}

Keyword: Knowledge-based Fault diagnosis

\section{Introduction}

The application of the weapon system is divided into two areas: the maneuvering field which moves the vehicle in the open fields and rough roads and the fire power field which manages the target by aiming the gun and cannibalize the enemy. Each area is a complex system with a combination of the components and the modules. In the military area, it is very important to recognize the fault in advance since some fatal defect of system causes the irreversible results. In this paper, the inference system for fault diagnosis is introduced. By analyzing the mechanical defects of the unit system or component / module of such a weapon system, it is possible to judge whether the system is defective, to determine the operational status of the system, and to take appropriate measures to occupy the advantage in the future battlefield environment.

In this paper, a fault diagnosis platform is constructed with the gun / turret drive system which is the typical system in the fire power field and based on the decision logic in the main controller. Based on this, it is possible to extract fault characteristic data and to perform fault diagnosis in various environments. The fault diagnosis platform consists of three levels: information processing, learning, and fault diagnosis. It sets the risk factors for the turret driving through preconception and contextual knowledge and performs fault diagnosis using the extracted feature data. In the fault diagnosis platform, the information processing level extracts the control data values of the components/ modules used in the system, and the learning level serves to connect the upper and lower levels. Finally, the fault diagnosis level detects the fault through inference. 


\section{Knowledge-based fault diagnosis}

There are many studies that introduces fault diagnosis method using explicit expressible inference concept of formal structure for shared concept about fault diagnosis using knowledge [1], a research that expresses the correlation of components and positions using feature data that is a risk factor [2]. In this paper, we present a fault diagnosis method using symbol representation of knowledge base and matching of representative values.

\section{Construction of Fault Diagnosis System using Symbol Representation}

Figure 1 shows the proposed knowledge based fault diagnosis structure. The proposed system is composed of three levels, and it is constructed to diagnose through inference by expressing the characteristic value possessed by each component at the fault diagnosis level by symbols, and the learning level is organically connected between the upper and lower levels. The information processing level is structured to extract feature values from the controller that controls the component unit [3].

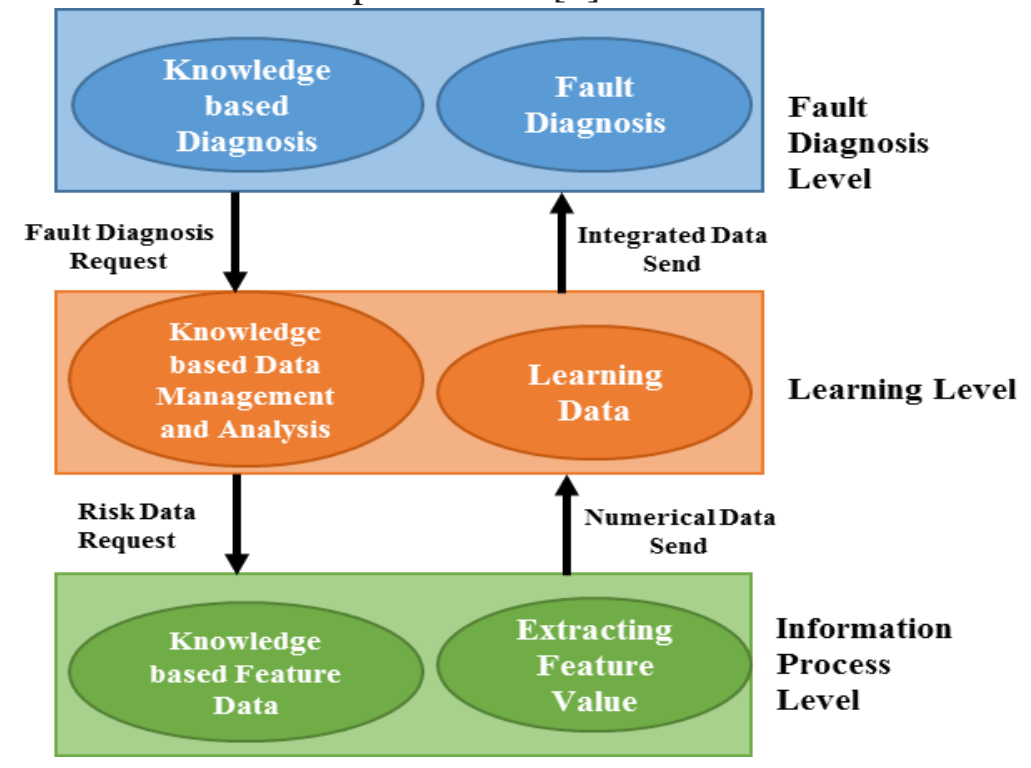

Figure 1: Knowledge based Fault Diagnosis Structure

Figure 2 shows the flow chart of fault diagnosis system. In order to diagnose faults, a symbol to be diagnosed is selected based on the device name or component, the symbol descriptor is generated in a semantic language, and the fault diagnosis level is requested to the learning level. The learning level selects data whose priority is predicted based on the severity, occurrence frequency, and detectability based on the data representing the requested symbol at the fault diagnosis level, and transmits and receives characteristic values to the information processing level. The learning DB (Database) learns how to prioritize fault-tolerant components as well as the life of the equipment. The information processing level extracts feature values determined to be risk factors and transmits them to the learning level. The transmitted feature values are matched and analyzed with the semantic language, and the similarity is measured at the fault diagnosis level to perform the fault diagnosis. 


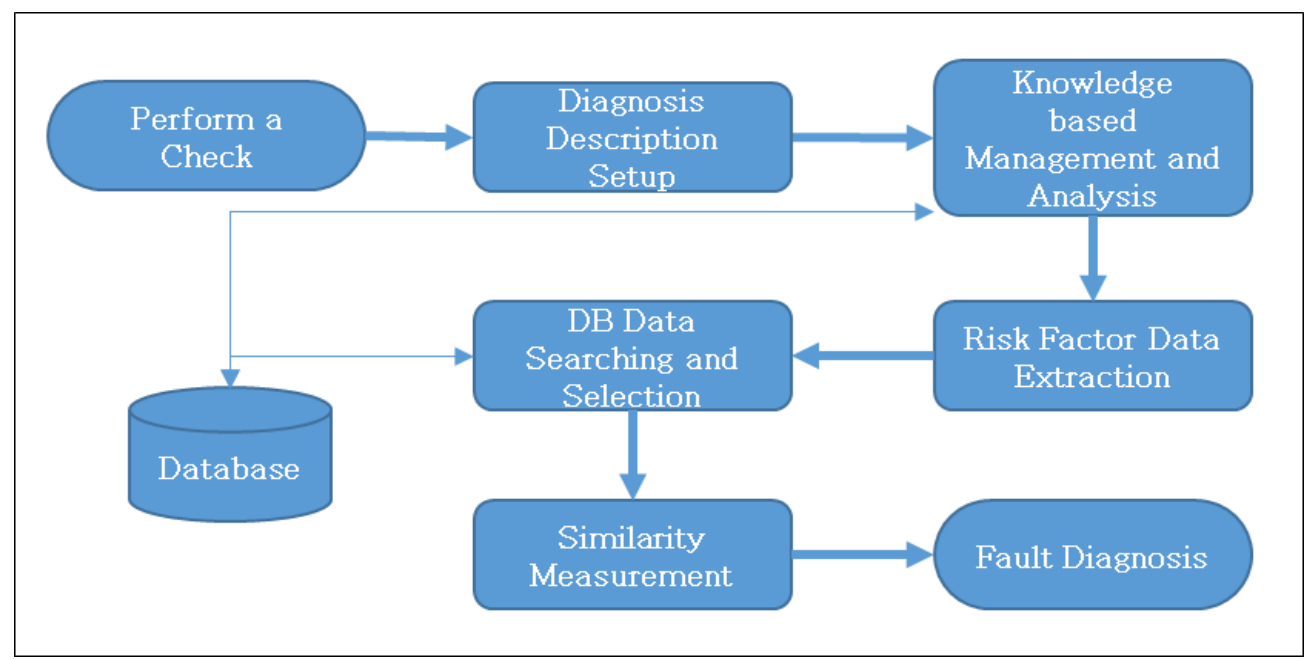

Figure 2 : Flow chart of fault diagnosis system

\section{Process for troubleshooting}

The fault diagnosis level sets the symbol used to represent the fault diagnosis. Symbols can represent generally used objects in a hierarchical structure. This structure consists of symbol representing the object itself and symbols representing the characteristics and characteristics of the object. Symbols representing the properties and characteristics of objects are called descriptors. These technologists mainly focus on concepts that express objects well among various concepts as show in Figure 3.

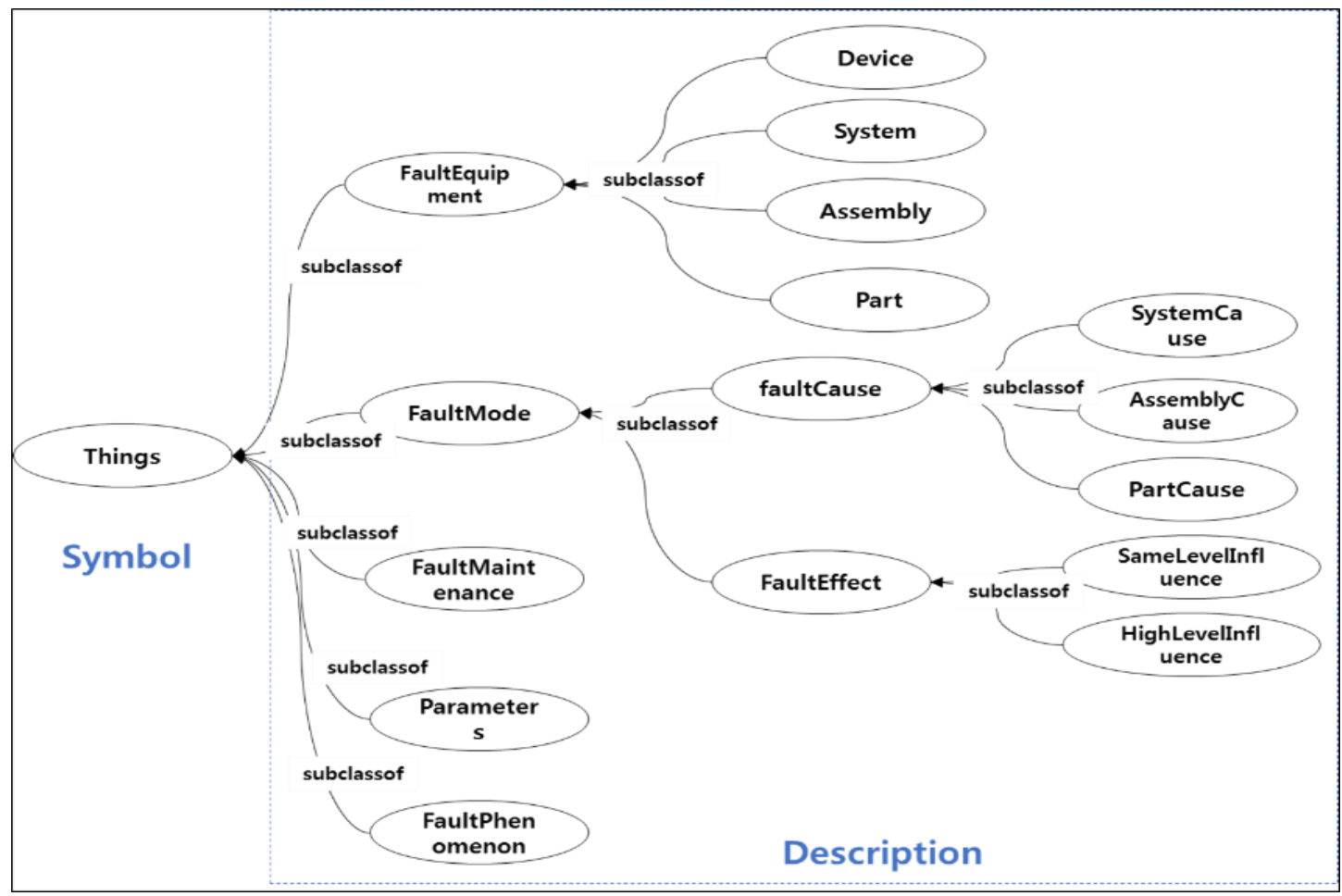

Figure 3 : Symbol Defined for Fault Diagnosis

As shown in Table 1, a properties of each object have its own domain and scope. Object properties are the simplest form of object property expressions, and inverse object properties allow for bidirectional navigation in class expressions and axioms [4]. 
Table 1 : Define the object properties of the class

\begin{tabular}{ccccc}
\hline No & Object Property & Domains & Ranges & $\begin{array}{c}\text { Inverse } \\
\text { Property }\end{array}$ \\
\hline 1 & stemFrom & FaultMode & Parameters & leadTo \\
\hline 2 & causeIs & FaultMode & FaultMode & N/A \\
\hline 3 & belongTo & FaultEquipment & FaultEquipment & N/A \\
\hline 4 & toEffect & FaultMode & FaultMode & N/A \\
\hline 5 & toSameLevelEffect & FaultMode & FaultMode & N/A \\
\hline 6 & toHighLevelEffect & FaultMode & FaultMaintenance & N/A \\
\hline 7 & offerInformation & FaultEquipment & FaultMaintenance & N/A \\
\hline 8 & toMaintenance & FaultMaintenance & FaultMode & maintenanceBy \\
\hline 9 & happenAt & FaultMode & FaultEquipment & hasFault \\
\hline 10 & hasPhenomenon & FaultMode & FaultPhenomenon & originateFrom \\
\hline
\end{tabular}

Once the attributes of the object are defined and the relationship of the data is established, a fault diagnosis structure is constructed as shown in Fig 4. The learning level step is to parse the data of the fault type priority of the system based on the FTRPN (Fuzzy-Time Risk Priority Number), store it in the database, and diagnose the fault through the query.

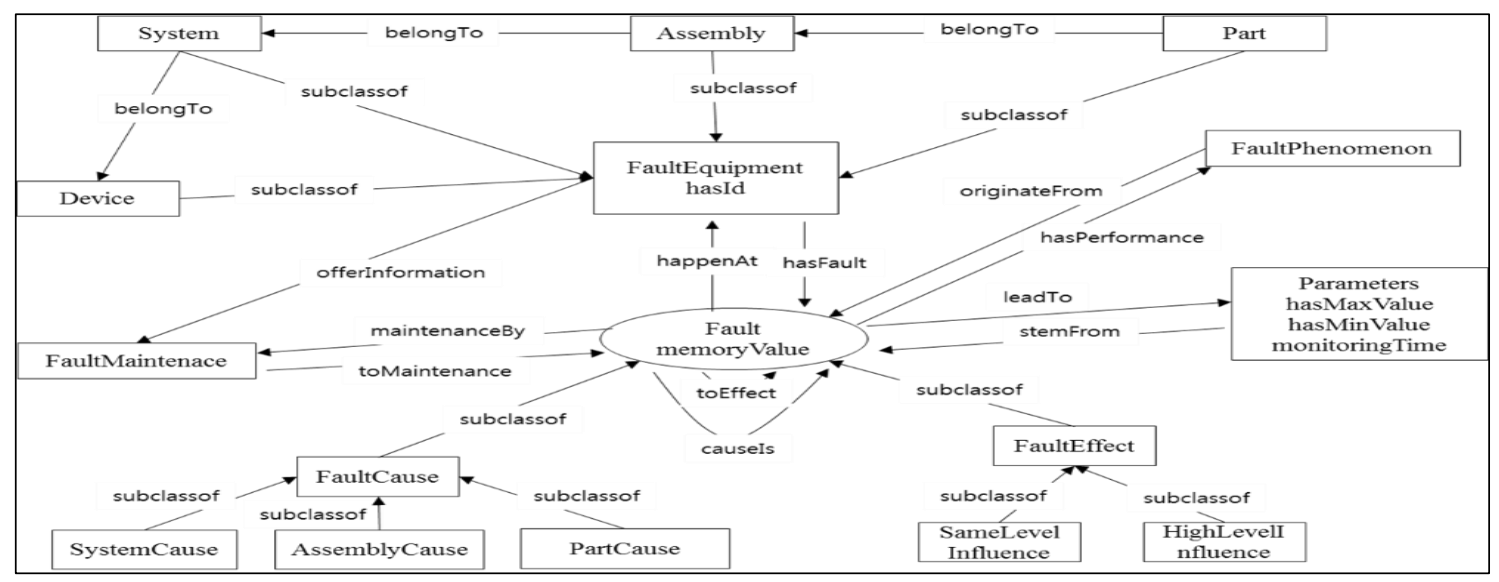

Figure 4 : Fault Diagnosis Structure

If a priority is determined for each fault type in the learning level, fault diagnosis and prediction can be performed using the correlation expression set in the descriptor of the symbol through ranking (priority) information.

Table 1: Fault diagnosis similarity measure

Table 2: Fault check result

\begin{tabular}{|c|c|c|}
\hline FTRPN & Ranking & Symbol \\
\hline$(57$ 9) $(7 \quad 1010)$ & $7 \sim 10$ & High \\
\hline$(357)$ & $4 \sim 6$ & Medium \\
\hline$(0 \circ 3)(135)$ & $1 \sim 3$ & Low \\
\hline
\end{tabular}

\begin{tabular}{|c|c|}
\hline Symbol & Description \\
\hline High & Need Replacement \\
\hline Medium & Need Check \\
\hline Low & Stay Operation \\
\hline
\end{tabular}

The learning level collects and analyzes the risk factor data of the information processing level and establishes correlation with the similarity measurement descriptor of the fault diagnosis level. Although FMEA(Failure Mode Effects Analysis) is a useful tool 
for evaluating reliability and failure modes, it is vulnerable to risk assessment and reliability evaluation because it is difficult to determine relative importance if different risk modes have the same risk priority value. In the learning level, the method for determining the risk priority of the fault type (1) and the weighting of the occurrence frequency based on the time accumulation degree (2) were used as show in Table 2, 3 [5].

IF $S_{i}$ is $F S_{i}^{m}$ and $O_{i}$ is $F S_{i}^{m}$ and $D_{i}$ is $F S_{i}^{m}$ THEN $Y_{i}$ is $F O_{i}$

FTRPN $=T * R P N * \omega=\sum_{i=0}^{n}\left(\frac{S_{i}}{100}\right) * R P N *\left(\sum_{i=1}^{n} \frac{\mathbb{}\left(\left\{\prod_{j=1}^{3} F S_{j}^{I}\left(x_{j}\right)\right\} * O_{i} \rrbracket\right)}{\sum_{i=1}^{n} \mathbb{L}\left\{\left\{\Pi_{j=1}^{3} F S_{j}^{I}\left(x_{j}\right)\right\} \rrbracket\right)}\right)$

It is possible to determine the life cycle of parts and systems by weighting over time and determine the fault type priority of the system when weighing the fuzzy generation rules and the occurrence frequency. The information processing level is used to extract the hazard data from the components and the controller and send it to the upper level of the knowledge base to analyze the fault impact and diagnose the fault. Through the data identified by each device, status data is obtained that can be used to determine whether the device is malfunctioning during equipment operation

\section{Test results}

A prior and contextual knowledge based fault diagnosis system was applied to the turret operation system. The cause of the fault is extracted from the information processing level by the component unit of the remote controller equipment and the priority result data of the fault type is determined at the learning level. The fault diagnosis level identifies the cause of the fault and judges whether or not the system is in continuous operation as shown in Fig 5.

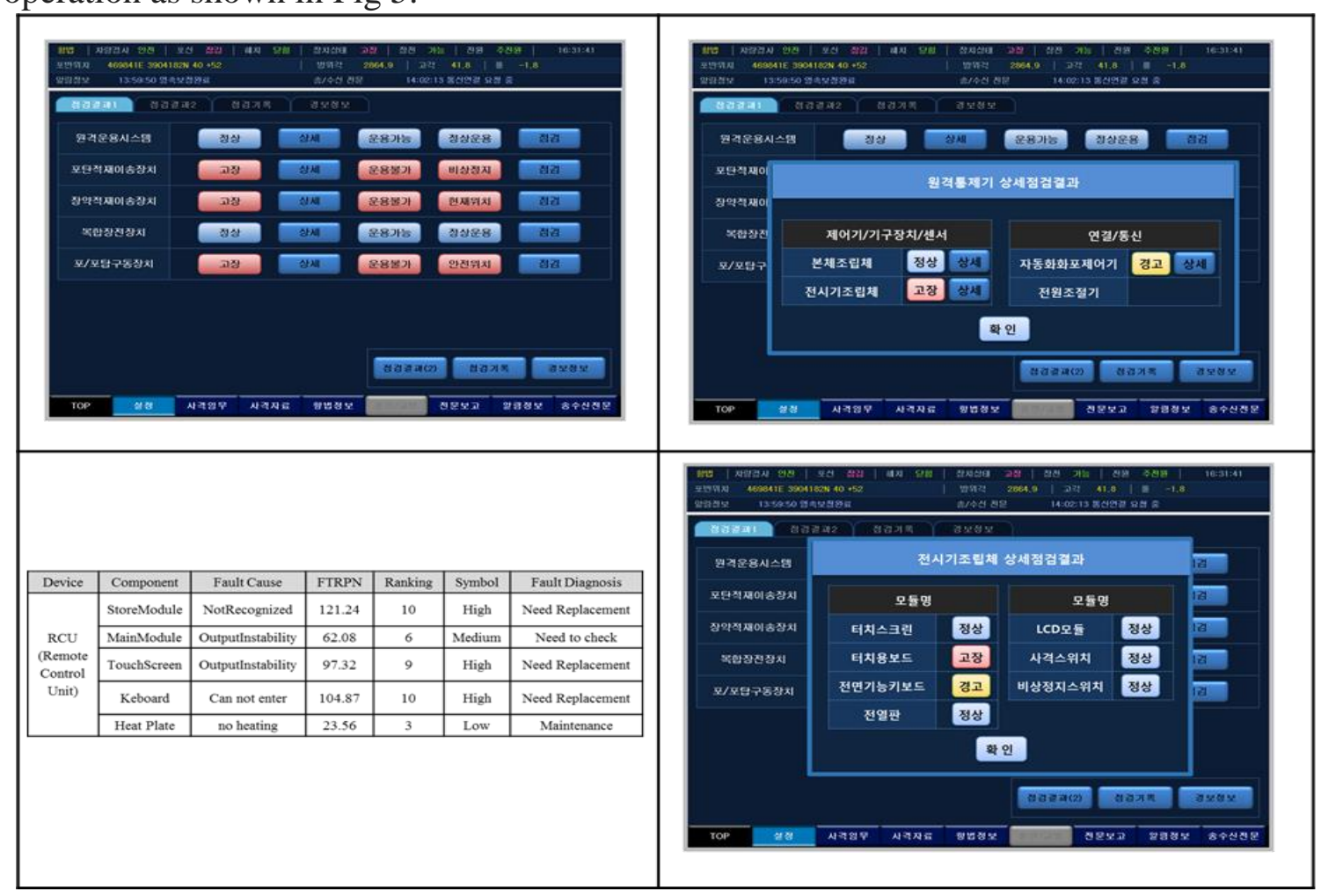

Figure 5 : UI Display for fault diagnosis and test result 


\section{Conclusion and future research}

In this paper, we propose an effective fault diagnosis technique based on the prior and contextual knowledge to effectively perform fault diagnosis of the turret / turret drive system. The test results show it is possible to predict and diagnose the cause of the complicated defects accurately by applying the prior and situational knowledge techniques rather than merely numerical data base. Through concrete design verification, we plan to derive reasoning system more robustly in dynamic environment.

\section{Reference}

[1] Park Yong-Tack, Construction of Multimedia Knowledge-based Systems, KOSEF, 1997, 951-0906-056-2

[2] CahnkgMo Jung, MyungChun Lee, Logistic Supportability Improvement Program for the Future Main Battle Tank. Journal of the Korea Society of Systems Engineering 1.2. 2005.7

[3] Gerevini A. and Nebel B., Qualitative Spatio-Temporal Reasoning with RCC-8 and Allen's Interval Calculus: Computational Complexity, In Proc. Of the $15^{\text {th }}$ European Conf. on Artificial Intelligence, 2002

[4] Feixiang $\mathrm{Xu}$, Xinhui Liu, Wei Chen, Chen Zhou and BingweiCao. Ontology-Based Method for Fault Diagnosis of Loaders. Mdpi Sensor 2018,18,729; doi:10.3390/s18030729

[5] Gyeongdong Baek, Sungshin Kim, Seongpyo Cheon, Heungwon Suh and Daehyung Lee, Prioritizing for Failure Modes of Dynamio Positioning System Using Fuzzy-FMEA. Journal of Korea Institute of Intelligent Systems, 2015, 25, 2, 174-179 